\title{
Neuroethics, Painience, and Neurocentric Criteria for the Moral Treatment of Animals
}

\author{
SHERRY E. LOVELESS and JAMES GIORDANO
}

\begin{abstract}
Neuroscience affords knowledge that can be leveraged in the ontological valuation of individuals, groups, and species. Sociocultural sentiments, norms, and mores may impede embracing such knowledge to revise moral attitudes, ethics, and policies. We argue that the practices of neuroethics will be valuable in that they ground ethico-legal discourse in (1) naturalistic philosophy; (2) the current epistemological capital of neuroscience; (3) the issues, problems, and solutions arising in and from neuroscientific research and its applications; and 4) the use of neurocentric criteria — such as painience - to define and resolve ethical decisions regarding attitudes toward and treatment of nonhuman animals.
\end{abstract}

Keywords: neuroethics; animals; animal welfare; pain; neurocentric criteria

\section{Neuroscientific Advancements and Attendant Responsibilities}

Neuroscience has enabled considerable opportunities to assess and define mechanisms of sensation, cognition, emotions, and behaviors. This has engendered a view of neural systems - and their functions - as embodied within organisms in environments. Understanding the neural bases of organisms' ecological interactions has twofold value: first, it fosters insight into the neurocognitive capabilities of individual beings and various species, and second, it prompts deeper reflection and more thorough address of what such functions and capacities mean relative to the ways that organisms are regarded and treated..$^{1}$ Indeed, history has revealed the use of various forms of neurocentric criteria to shape categorical distinctions regarding what types of organisms, and which individuals, may claim particular social goods. ${ }^{2,3,4}$ The scope and pace of contemporary neuroscientific advancement(s) have only fortified this trend, in part through heuristics that have been influential to establishing theories of mind.5,6 These heuristics are paradigmatically valid and of value, but it is important to note that a theory of mind can be a powerful tool with which to leverage beliefs, values, and behaviors, and with this power comes considerable responsibility. $7,8,9,10$

Much of the information gained from neuroscientific research has been derived from animal models, and there is building evidence to support that many animal species-for example, mammals, several species of vertebrates, and cephalopodshave nervous systems that are structurally and functionally sophisticated enough to sustain relatively complex cognitive processes. ${ }^{11,12}$ This challenges long-held

This work was supported, in part, by the J. W. Fulbright Foundation (JG); the William H. and Ruth Crane Schaefer Endowment (JG); and funding from the Center for Neurotechnology Studies of the Potomac Institute for Policy Studies (SEL), from the Human Science Center of the Ludwig-Maximilians Universität, Munich, Germany (JG), and from the Animal Behavior and Conservation Program, Department of Psychology, Hunter College, City University of New York, New York (SEL). The authors acknowledge the assistance of Rachel Landsberg on this project. 
ideas about animals' incapacity for consciousness, feeling pain, and experiencing emotions and, more broadly, contests anachronistic, if not wholly dogmatic, philosophical distinctions between humans and animals. ${ }^{13,14}$ As the philosopher Fritz Jahr has noted, "scientific triumphs . . . have taken away the dominant position of the human being in the world in general. Philosophy, formerly prescribing leading ideals for the natural sciences, now has to build her systems on the basis of scientific knowledge from the natural sciences."15 Of course, differing epistemological and anthropological positions exist, and there is ongoing debate about the merit of specific neurocentric criteria as a basis for ontological status and ethical conduct, and if-and which-neurocognitive characteristics are (and should be) important for the moral and legal treatment of animals.

Our position is that a deepening understanding of the ways that nervous systems and brains are involved in (or evoke) those characteristics that are valued in individuals, groups, and a species should compel and sustain the ways that the organisms that possess such characteristics are regarded and treated. But, we also note that embracing such knowledge to revise moral attitudes and the ethics and policies that affect nonhuman animals will not be simple or easy, given particular sociocultural sentiments, traditions, norms, and mores. We believe that the relatively new field of neuroethics will be valuable to address these challenges in that it grounds ethico-legal discourse in (1) a naturalistic philosophy; (2) the current epistemological capital of neuroscience; (3) the moral issues, problems, and solutions arising in and from neuroscientific research and its applications; and (4) the use of neurocentric criteria to define and resolve ethical questions and decisions. ${ }^{16,17,18,19,20}$

\section{Painience-A Minimum Neurocentric Criterion}

The recognition of (certain species of) animals as being sentient as a basis for and the establishment of ethical and legal codes for animal welfare ${ }^{21,22,23}$ represents an explicit departure from arbitrary speciesism, and a noteworthy advancement toward the employment of neurocentric criteria. However, despite progress in neuroscience and neurotechnology, much remains unknown about the nature of sentience-for instance, the shifting definitions of "higher" and "lower" consciousness, the neurocognitive function(s) of animals, what species of animals are sentient, and if and what particular properties of sentience should be relevant to discern the extent of moral regard and the level of good that should be afforded. ${ }^{24}$

Hence, we propose that an organism's capacity to feel pain as noxious (injurious) and explicitly hurtful (that is to say, pain qua pain, not simply nociception but nociperception) represents a minimum criterion on which to base and predicate moral consideration and actions. ${ }^{25,26}$ But this too, is not without complication. The formal definition of pain as "an unpleasant sensory and emotional experience"27 obtains that for an objective neural event to be subjectively experienced as pain (and not merely a high-intensity, aversive stimulus), it would necessarily involve the qualities of sensation, cognition, and emotion. To be sure, pain is a multidimensional event that is "a sensation in a part of the body . . . and an emotional experience." 28 It has been claimed that animal pain is not as significant as that of humans, given that certain areas of the cortex and neural pathways that have been shown to be responsible (in humans) for the associative linking of sensations to cognition and emotion are not present in all animals. ${ }^{29,30,31}$ 
We object to this claim for several reasons. First, this position fails to acknowledge the most current perspective(s) regarding the structure-function relationship of nervous systems; these can be colloquially summarized using a computer analogy to posit that the presence of the "wetware" (the neural substrates) is often a reliable predictor that the "program" (pain) can and will be executed. ${ }^{32}$ This is strengthened by comparative studies of mammalian species as anatomical, physiological, and behavioral models of human development, neurophysiology, and pain. ${ }^{33}$ Perhaps what is more important is the recognition that the experience of pain, while involving a number of higher neuroanatomical loci-for example, the somatosensory and associative cortices-is also reliant on subcortical structures, which are engaged as hierarchical networks and contribute to mental processes. ${ }^{34,35,36,37,38}$ Thus, the second flaw in arguments that attempt to negate the experience and profundity of animal pain is that they tend to attribute the function of networked hierarchies of neural structures (if not the whole brain) to particular parts-what has been called the "divisional" or mereological fallacy. ${ }^{39}$

Third, one-to-one representation of neural structure-functional relationships between humans and animals need not be present for the occurrence of cognitive, emotional, and behavioral processes. The networks involved in pain processing are not necessarily identical, that is to say, homologous, to those of humans but instead may-and often do-function analogously to conjoin distinct neural substrates to obtain the pain event and experience. This speaks in return to the second point-namely, that brains are embodied within an organism, and this individual neural structure and function gives rise to differing subjective experiences.

To reiterate, although the phenomenology of pain cannot be objectively measured in animals — including the human animal ${ }^{40,41}$ — the presence and activity of physiological systems shown to process painful stimuli can lead to strong inference(s) regarding accompanying and resultant unpleasant emotional experience. So, although it is possible, and in some cases likely, that the perception and meaning of animal pain is different from that of humans (given distinctions in ecology, lived body, and lifeworld), it is important to acknowledge that neural systems are in place and operational that allow many animal species to experience such stimuli as noxious, and this noxiousness is fundamental to the cognitiveemotional event and hurtful nature of pain.

But, at present, we cannot know the phenomenological characteristics of animal pain, and although an understanding of various organisms' nervous systems' capacity to subtend pain is necessary and important, argument by analogy could also support the value and use of behavioral expressions-such as increased vocalizations, agitation, writhing, reluctance to move and decreased locomotion, attending to and guarding parts of the body, and huddled postures-as indicative of pain. ${ }^{42}$ In mammals, such behaviors have been shown to represent more than merely "pseudoaffective" responses and are now regarded as manifestations of cognitive and emotional processes, abstract dimensions of fear, and (some form of) suffering. ${ }^{43,44,45}$

However, attending, guarding, and writhing responses have also been identified in crustaceans, certain insects, and other invertebrates. ${ }^{46,47}$ Additionally, it has been shown that these creatures have nocisponsive nerve endings, specialized nociceptors, and neural pathways to subserve high-intensity noxious stimuli. ${ }^{48}$ Taken together, these findings would allow the possibility that such organisms experience at least nociception, and some type of nociperception 
may also be plausible. Therefore, we claim that recognition of the neuroscientific fact that pain can occur in a particular organism is a necessary and sufficient condition to afford that organism basic moral consideration and respectful treatment, that is to say, to avoid the purposeful infliction of pain when and where possible. This is consistent with a number of ethical traditions that base human action on premises of nonharm and/or some version of a "golden rule" maxim ("do unto others ...") that begins from a presumption that said others can experience "hurt" in ways similar or analogous to the way(s) that humans know it. ${ }^{49,50,51}$ To take this argument a step further, the obligation to exercise such consideration reflects a view that is derived from the existential vulnerability of painient nonhuman animals and that positions them as the subjects of human responsibility and care. $.52,53,54$

\section{Ryder's Painism Redux}

In this context, we hold that Richard Ryder's thesis of "painism" offers a number of precepts, which Ryder called "rules," for the prevention and treatment of pain that may be worthwhile to a neuroethical approach to the moral regard and care of animals. ${ }^{55}$ Many of Ryder's more social assertions (about such things as politics, for example) have been disparaged as ampliative, and a complete description, discussion, and debate of Ryder's manifesto are beyond the scope of the present essay. ${ }^{56}$ However, we believe that it would be erroneous to proverbially discard the baby with the bathwater, as Ryder's core thesis defines the nature and purpose of morality and ethics as relative and relevant to pain and the painient. Although his statement that pain and suffering represent the "only evils" 57 may be extreme when considering the human condition, a number of harms within the lifeworld of animals-particularly as affected by humansinvolve, or are directly or indirectly related if not attributable to pain and its effects. The imperative to recognize animals' capacity to experience pain, and the human responsibilities that arise thereupon are obtained in Ryder's claim that "moral standards should apply equally to all painient individuals, regardless of species." 58

From these fundamental premises follow proscriptions against the infliction of "unconsented pain" and pain "for trivial purposes." ${ }^{59}$ We maintain that these support responsibility for the stewardship of both the most contemporary knowledge of neural mechanisms of animal pain and the power to inflict, prevent, and/or mitigate pain, which undergird the ethical treatment of animals, in general. Explicit to these statements is the importance of a more finely grained analysis-and revision-of the ways that animal welfare is imparted in various utilitarian agendas of human society.

Although we pose this argument as a basis for both individual reflection and to contribute to guidelines and policies for the treatment of animals, we are not naïve to, nor do we deny, human needs and motives for flourishing. ${ }^{60}$ Nor is this a call for strict veganism, the abandonment of animal products, or a prohibition against pets, zoos, and the use of service and work animals. We do not exhort extremism in the moral consideration and treatment of animals. But, to paraphrase scientist and animal welfare advocate Temple Grandin, the more science learns about animals, the more we must develop and institute sociocultural considerations of, and protections for, their welfare. ${ }^{61}$ 


\section{Pain Research Involving Animals}

This spirit of scientific inquiry generates a potential conundrum: given that research is the principal tool that enables the articulation of science and acquisition of knowledge, there is a rational and defensible argument for continued studies to further elucidate the neurocognitive functions and capabilities of animals. Yet, the goals of such investigations, how such studies are undertaken, and how findings are utilized are equally important if science is to remain ethically sound. Pain research involving animals provokes particular ethical discussion and controversy because it axiomatically is intended to elicit pain in the research subject, and a full presentation of this discussion exceeds the limits of this article. ${ }^{62}$

Animal studies also have been-and remain-important to guide and complement investigations involving human subjects. ${ }^{63}$ However, a number of limitations and criticisms of animal models of pain have been brought to the fore, including a lack of ecological validity, narrow emphasis on sensory events, inadequacies when addressing the epidemiology of chronic pain (in both animal and human populations), and inferiority of design and reporting standards. ${ }^{64}$ Identifying these gaps and inadequacies is crucial to the iterative reappraisal of the scope, conduct, and value of pain research in animals (if not animal research overall). Questions essential to such reappraisal reflect the well-known "Three Rs" (reduction, revision, and replacement), as proposed by Russell and Burch, and ask (1) whether painful methods can be replaced with other, less invasive protocols, (2) if and how protocols and methods can be refined to minimize the painful nature of the research, and (3) whether and how the numbers of animals - and perhaps the overall number of studies-can and should be reduced. ${ }^{65}$

Furthermore, there is continued uncertainty and debate within the scientific community as to whether or not animals provide a realistic model of human pain. $66,67,68,69$ This reflects a type of "extrapolation dilemma"70 that elicits two potential scenarios that are important to the ways that results from research studies are used. In scenario A, the model is "distant" and therefore, although it provides some information, remains "insufficient" for direct comparative applications due to differences and gaps in the structures and functions of systems studied. The resulting implication is that we should abandon the model in favor of others that are more proximate and applicable. In scenario B, the model is "proximate" and therefore "sufficient" given the similarity of structures and functions of those systems studied to the comparative target. The resulting implication is that information from the model should be applied both to the comparative target and to the model. If we (rightly) assume that current animal models of pain fulfill criteria of being proximate and sufficient sources for information that may be directly relevant, and that may be translated to knowledge about human systems, then what is learned from studying animals-to gain information about humans - is applicable to both humans and (at least) those species of animals that are the object of study. If this is so, then the good of research could be maximized through the application and use of such knowledge to inform and direct subsequent actions toward both humans and animals.

From this, we posit that a foundational stance for the conduct of animal research and care could be derived from, and based on, a version of Rawls's principle of maximin: given (1) the unequal distribution of knowledge, capability, and power between humans and animals and (2) a reasonable expectation that any organism 
with the anatomical and physiologic capabilities for pain will manifest this experience, then (3) ethical probity can be achieved and sustained by maximum prevention and mitigation of such pain, as a minimum benefit that should be allocated by those in power toward the provision of the welfare of those organisms over whom such power is exercised. ${ }^{71}$

\section{A Path Forward}

We recognize the potential benefit of pain research in animals, but here again we question how, and to what effect, research findings will be utilized. We believe that in light of knowledge about pain gained from such investigations to date, continued pain research in animals is acceptable if and only if any and all such studies (1) adhere to practical and ethical guidelines that are consistent with the most contemporary understanding of comparative and cognitive neuroscience, (2) adopt a frank precautionary stance when the welfare of any painient creature is at risk, and (3) attempt to directly employ research findings to benefit animal species through reciprocal and/or reverse translation, writ both small, within veterinary practice, and large, as applied to the care and treatment of wild, captive, livestock, and domestic animals. ${ }^{72}$

Although we offer painience as a minimum criterion for neuroethical regard, this is but a first step, as we anticipate that further understanding of animal consciousness, emotions, and higher cognitive functions will instantiate stricter moral standards for animal research and welfare. These neuroethical premises are not merely confined to the laboratory but extend into the public sphere to affect social conduct as well. We maintain that an important element in the social acknowledgement and embrace of new concepts will be the development of neuroethically informed and neuroethically based programs of primary, secondary, and postsecondary education. Such programs would inculcate more meaningful apprehension of the ways that neuroscience may foster a non-anthropocentric appreciation of animals and would strengthen the notion of human/animal differences as distinctions of degree, rather than natural kind, and in so doing weaken anachronistic dualisms that have guided human treatment of animals. This will be evermore necessary as (1) society becomes more neuroscientifically and technologically capable, dependent, and oriented; (2) neuroscience and technology become increasingly powerful forces of social construction and/or deconstruction; and (3) humanity confronts the growing, incumbent responsibility to use such knowledge and technology in ways that are morally sound. ${ }^{73}$ Furthermore, neuroscientific information and neuroethical precepts should inform and influence policies and laws directing and governing the treatment of animals.

Although neurocentric criteria can be seen as novel and important grounds for animal welfare and protection legislation, the statutory protection of animals is not new. The first formal laws to assert animal welfare were enacted in Germany in the late 1700s, followed in 1822 by Martin's Law being signed into effect in Britain. ${ }^{74}$ Regional German statutes during the early 1800 s were superseded by the first encompassing national animal protection law in 1871, which was strongly influenced by both the philosophical worldview and the expanding scientific empiricism of the time. ${ }^{75}$ Ethically based, comprehensive national animal protection laws were ratified in Germany during the 1920s, and these became the basis 
for the German Law on Animal Protection (Reichstierschutzgesetz), first passed in November 1933, and subsequently expanded in $1938 .{ }^{76}$ Following the fall of the Third Reich, the law was amended during the 1950s and 1960s and finally rescinded in 1972, in favor of a more contemporary iteration - the Tierschutzgesetz-which maintained the ethical foundations of the earlier versions but entailed greater appreciation for the physical and psychological lives of animals and obtained a more balanced view of animal and human interests. Although largely rhetorical, the law fostered the incorporation of cohesive animal protection language into German civil codes and state constitutions and provided the impetus for a number of attempts at fortification through national constitutional amendments during the mid-1990s. ${ }^{77}$ These efforts ultimately culminated in the 2002 addition of a clause within Article $20 \mathrm{a}$ of the German constitution (viz., "und die Tiere," or "and the animals") ${ }^{78}$ that ensured regard and protection of animals in the formulation and enactment of all laws and judicial decisions. ${ }^{79}$ Moreover, the trend in German law since the 1970s has been important to animal protection statues in other European countries, including Austria and Switzerland. ${ }^{80}$ And although animal protection regulations throughout the European Union (as well as the United States and Canada) ${ }^{81}$ are generally not as extensive or stringent as in Germany, ${ }^{82}$ the Tierschutzgesetz concept of epistemologically informed, ethically based regard and treatment of animals may be seen as setting the stage for international regulations, such as the 2009 Treaty of Lisbon, which amended stances on animal welfare provided by the Treaty on European Union, and the Treaty on the Functioning of the European Union, and in so doing established animal protection as a fundamental ethico-legal principle..$^{83}$

In conclusion, our aim is not to prescribe a form of neuroethical absolutism, for we acknowledge that individuals' moral compasses differ, and cultural norms and mores vary. But ethical analysis and sound legal deliberation must begin from fact(s), and it is our hope that neuroscientific information about the painience of other species will dispel dated apologia for the mistreatment of animals and foster a sense of awareness and responsibility to inform and guide the ethical reflections, choices, and actions of both individuals and societies.

\section{Notes}

1. Giordano J, Benedikter R. Neurotechnology, culture, and the need for a cosmopolitan neuroethics. In: Giordano J, ed. Neurotechnology: Premises, Potential, and Problems. Boca Raton, FL: CRC Press; 2012:233-42.

2. Aristotle. Nicomachean Ethics. Book VI. Ross D, trans. London: Oxford University Press; 1966.

3. Galton F. Inquiries into Human Faculty and Its Development. New York: AMS Press; 1973.

4. Sorabji R. Animal Minds and Human Morals: The Origins of the Western Debate. Ithaca, NY: Cornell University Press; 1993.

5. Gigerenzer G, Goldstein DG. Mind as computer: Birth of a metaphor. Creativity Research Journal 1996;9(2-3):131-44.

6. Giordano J. Neurotechnology as demiurgical force: Avoiding Icarus' folly. In: Giordano J, ed. Neurotechnology: Premises, Potential, and Problems. Boca Raton, FL: CRC Press; 2012:1-14.

7. Giordano J, Benedikter R, Kohls NB. Neuroscience and the importance of a neurobioethics: A reflection upon Fritz Jahr. In: Muzur A, Sass H-M, eds. Fritz Jahr and the Foundations of Global Bioethics. Münster, Berlin: LIT Verlag; 2012.

8. Giordano J, Benedikter R. An early—and necessary—flight of the Owl of Minerva: Neuroscience, neurotechnology, human socio-cultural boundaries, and the importance of neuroethics. Journal of Evolution and Technology 2012;22(1):110-15.

9. Giordano J, Olds J. The interfluence of neuroscience, neuroethics and legal and social issues: The need for (N)ELSI. American Journal of Bioethics-Neuroscience 2010;1(4):12-14. 
10. Giordano J. Neuroethics_coming of age and facing the future. In: Giordano J, Gordijn B, eds. Scientific and Philosophical Perspectives in Neuroethics. Cambridge: Cambridge University Press; 2010.

11. Page G. Inside the Animal Mind: A Groundbreaking Exploration of Animal Intelligence. New York: Doubleday; 1999.

12. Griffin DR. Animal Minds. Chicago: University of Chicago Press; 1994.

13. See note 12, Griffin 1994.

14. White T. In Defense of Dolphins: The New Moral Frontier. Oxford: Wiley-Blackwell; 2007.

15. Jahr F. Bio-Ethik: Eine Umschau über die ethischen Beziehungen des Menschen zu Tier und Pflanze. Kosmos. Handweiser für Naturfreunde 1927;24(1):2-4, at 4.

16. See note 9, Giordano, Olds 2010.

17. Giordano J. Integrative convergence in neuroscience: Trajectories, problems and the need for a progressive neurobioethics. In: Vaseashta A, Braman E, Sussman P, eds. Technological Innovation in Sensing and Detecting Chemical, Biological, Radiological, Nuclear Threats and Ecological Terrorism. NATO Science for Peace and Security Series. New York: Springer; 2012.

18. Giordano J. Neuroethics: Traditions, tasks and values. Human Prospect 2011;1(1):5-10.

19. See note 7, Giordano et al. 2012.

20. Giordano J. Advances in Neurotechnology: Premises, Potential and Problems. Boca Raton, FL: CRC Press; 2012.

21. Turner J, D'Silva J, eds. Animals, Ethics, and Trade: The Challenge of Animal Sentience. London: Earthscan; 2006.

22. Bentham J. Introduction to the Principles of Morals and Legislation. Oxford: Clarendon Press; 1823.

23. Policy and cultural implications. Compassion in World Farming; available at http://ciwf.org.uk/ animal_sentience/policy_and_culture (last accessed 12 Feb 2012).

24. Frankena WJ. Beneficence in an ethics of virtue. In: Shelp E, ed. Beneficence and Health Care. Dordrecht: Reidel; 1982.

25. See note 10, Giordano 2010.

26. Giordano J. The neuroscience of pain, and the neuroethics of pain care. Neuroethics 2009;3(1):89-94.

27. International Association for the Study of Pain (IASP). IASP Pain Terminology; available at http:// www.iasp-pain.org/Content/NavigationMenu/GeneralResourceLinks/PainDefinitions/\#Pain (last accessed 20 June 2012); 2009.

28. See note 27, IASP 2009.

29. Lascelles BDX, Flecknell PA. Do animal models tell us about human pain? Pain: Clinical Updates 2010;XVIII(5):1-5; available at http:/ /www.iasp-pain.org/AM/AMTemplate.cfm?Section=HOME, HOME\&TEMPLATE $=/$ CM $/$ ContentDisplay.cfm\&CONTENTID=15073\&SECTION=HOME, HOME (last accessed 20 June 2012).

30. Whiteside GT, Adedoyin A, Leventhal L. Predictive validity of animal pain models? A comparison of the pharmacokinetic-pharmacodynamic relationship for pain drugs in rats and humans. Neuropharmacology 2008;54(5):767-75.

31. Peter Carruthers has asserted that animal pain is not as morally significant as human pain, which asserts a definitive speciesism based on philosophical grounds that are reflective, at least to some extent, of a form of natural law. Although we recognize the value of proximate relationships-see, e.g., note 60-we believe that the encompassing claim against the moral consideration of a painient creature's suffering at the very least fails to acknowledge grounding maxims of nonharm and at worst establishes arbitrary criteria for the determination of which organisms deserve moral regard and beneficent treatment. This latter point has been raised in critique of the divergent and contradictory attitudes and practices fostered by the 1933 and 1938 iterations of the Animal Protection Laws (Reichstierschutzgesetz) and Nuremburg Laws during the Third Reich. For further discussion, see Arluke A, Sax B. The Nazi treatment of animals and people. In: Birke L, Hubbard R, eds. Reinventing Biology. Bloomington: Indiana University Press; 1995:228-60. For a thorough philosophical counterpoint to Carruthers, see Singer P. In Defense of Animals. New York: Blackwell; 1985.

32. Although this is not to infer that we view the nervous system and/or brain "as" a computer, neural systems and brains are involved in computational processes. See, e.g., Kelso SJA. Dynamic Patterns: The Self-Organization of Brain and Behavior (Complex Adaptive Systems). Cambridge, MA: Massachusetts Institute for Technology; 1995, and Doya K, Ishii S, Pouget A, Rao RPN, eds. Bayesian Brain: Probabilistic Approaches to Neural Coding (Computational Neuroscience). Cambridge, MA: Massachusetts Institute of Technology; 2007.

33. Bekoff M. Cognitive ethology: The comparative study of animal minds. In: Bechtel W, Grahm G, eds. Blackwell Companion to Cognitive Science. Oxford: Blackwell; 1995. 
34. Salloway S, Cummings J. Subcortical disease and neuropsychiatric illness. Journal of Neuropsychiatry and Clinical Neuroscience 1994;6:93-9.

35. Edelman GM. Wider than the Sky: The Phenomenal Gift of Consciousness. New Haven, CT: Yale University Press; 2004.

36. Baars BJ. A Cognitive Theory of Consciousness. Cambridge: Cambridge University Press; 1998.

37. Steinke GK, Galan RF. Brain rhythms reveal a hierarchical network organization. Public Library of Science Computational Biology 2011;7(10):e1002207. doi:10.1371/journal.pcbi.1002207.

38. Kelso JAS, Fuchs A. Self-organizing dynamics of the human brain: Intermittency, antimonotonicity and Sil nikov chaos. Chaos 1995 Mar;5(1):64-9.

39. Bennett MR, Hacker PMS. Philosophical Foundations of Neuroscience. Oxford: Wiley-Blackwell; 2003.

40. Giordano J, Abramson KD, Boswell MV. Pain assessment: Subjectivity, objectivity and the use of neurotechnology part one: Practical and ethical issues. Pain Physician 2010;13(4):305-15.

41. This speaks to Thomas Nagel's existential question, "What is it like to be a bat?" reflecting the essential subjective and solely self-transparent qualities of consciousness. In the absence of some ability to explicitly communicate first-person experience, interpreting the phenomenological conditions of others becomes something of a hermeneutical exercise. Attempts to bridge the subjectivity-objectivity gap (e.g., through the use of neurotechnology) remain largely unsuccessful and are a focus of much of current pain and consciousness research; see Nagel T. Mortal Questions. Cambridge: Cambridge University Press; 1979; Giordano J. Pain: Mind, Meaning and Medicine. Glen Falls, PA: PPM Press; 2009;Giordano, J The neuroscience of pain, and the neuroethics of pain care. Neuroethics 2009;3(1):89-94; Giordano J, Benedikter R, Boswell MV. Pain medicine: Biotechnology, and market effects: Tools, tekne, and moral responsibility. Ethics in Biology, Engineering and Medicine 2010;1(2):133-40.

42. National Research Council. Guidelines for the Care and Use of Mammals in Neuroscience and Behavioral Research. Washington, DC: National Academy Press; 2003.

43. Sufka KJ, Weldon M, Allen C. The case for animal emotions: Modeling neuropsychiatric disorders. In Bickle J, ed. Oxford Handbook of Philosophy of Neuroscience. Oxford: Oxford University Press; 2009.

44. See note 42, National Research Council 2003.

45. There is considerable discussion and debate about putative distinctions between pain and suffering that may be important to definitions of painience and derivative moral and ethical implications. For a thorough discussion of defining characteristics and putative neural mechanisms of pain and/or suffering, see Moskovitz P. Understanding suffering: The phenomenology and neurobiology of the experience of illness and pain. In: Giordano J, ed. Maldynia: Multidisciplinary Perspectives on the Illness of Chronic Pain. New York: Taylor Francis; 2010.

46. Sherwin CM. Can invertebrates suffer? Or how robust is argument-by-analogy? Animal Welfare 2001;10:S103-18.

47. Eisemann $\mathrm{CH}$, Jorgensen WK, Merritt DJ, Rice MJ, Cribb BW, Webb PD, et al. Do insects feel pain?-A biological view. Cellular and Molecular Life Sciences 1984;40(2):164-7.

48. Fiala A. Neuroethology: A neuronal self-defense mechanism in fly larvae. Current Biology 2008; 13(3):R116-17.

49. Linzey A. Why Animal Suffering Matters: Philosophy, Theology and Practical Ethics. Oxford: Oxford University Press; 2008.

50. Gert B. Common Morality: Deciding What to Do. New York: Oxford University Press; 2007.

51. Pfaff D. The Neuroscience of Fair Play: Why We (Usually) Follow the Golden Rule. Washington, DC: Dana Press; 2007.

52. Kant I. Groundwork of the Metaphysics of Morals. Gregor M, ed. Cambridge: Cambridge University Press; 1998.

53. Ryder RD. Painism: A Modern Morality. London: Open Gate Press; 2001.

54. Singer P. Animal Liberation: A New Ethics for Our Treatment of Animals. New York: Random House; 1975.

55. See note 53, Ryder 2001.

56. For a more thorough address, see, e.g., Giordano J. Pain: Mind, Meaning and Medicine. Glen Falls, PA: PPM Press; 2009; Ingensiep HW. Painism-a new ethics: Richard Ryder's moral theory and its limitations. In: Giordano J, ed. Multi-Disciplinary Perspectives on the Illness of Chronic Pain. New York: Taylor-Francis; 2010.

57. See note 53, Ryder 2001, at 27.

58. See note 53, Ryder 2001, at 119.

59. See note 53, Ryder 2001, at 30. 
60. Of course, such consideration and treatment would need to be situated within an ethical framework of proximate value of kith and kin and an appreciation of the real risk of harms that may occur should pain be inflicted in an animal under these circumstances, e.g., an animal attack on one's self, child, spouse, etc.; see, e.g., Powers M. Contemporary defenses of the doctrine of double effect. Revue Internationale de Philosophie 1995;193:341-56; Regan T. Matters of Life and Death: New Introductory Essays in Moral Philosophy. 3rd ed. New York: Random House; 1993; Schofield M, Striker G, eds. The Norms of Nature. New York: Cambridge University Press; 1986.

61. Grandin T. Animals are not things: A view on animal welfare based on neurological complexity; available at http:/ / www.grandin.com/welfare/animals.are.not.things.html (last accessed 22 Jan 2012).

62. See, e.g., Regan T. Defending Animal Rights. Champaign: University of Illinois Press; 2001; Derbyshire SWG. Time to abandon the three Rs. Scientist 2006;20:23; Greek CR, Greek JS. Is the use of sentient animals in basic research justifiable? Philosophy, Ethics, and Humanities in Medicine 2010;5:14; Greek CR, Greek JS. Specious Science: Why Experiments on Animals Harms Humans. New York: The Continuum International Publishing Group; 2003.

63. Mogil JS, Davis KD, Derbyshire SW. The necessity of animal models in pain research. Pain 2010;151(1):12-17.

64. See note 63, Mogil et al. 2010.

65. Russell WMS, Burch RL. The Principles of Humane Experimental Technique. London: Methuen; 1959.

66. Bennett GJ, Xie Y- K. A peripheral mononeuropathy in rat that produces disorders of pain sensation like those seen in man. Pain 1988;33:87-107.

67. Mogil JS. Animal models of pain: Progress and challenges. Nature Review of Neuroscience 2009; 10:283-94.

68. Mogil JS, Crager SE. What should we be measuring in behavioral studies of chronic pain in animals? Pain 2004;112:12-15.

69. Rice ASC, Cimino-Brown D, Eisenach JC, Kontinen VK, LaCroix-Fralish ML, Machin I, et al. Animal models and the prediction of efficacy in clinical trials of analgesic drugs: A critical appraisal and call for uniform reporting standards. Pain 2008;139:241-5.

70. Steel D. Across the Boundaries: Extrapolation in Biology and Social Science. Oxford: Oxford University Press; 2007.

71. Rawls J. A Theory of Justice. Cambridge, MA: The Belknap Press of Harvard University Press; 1971.

72. We have referred to this expanded paradigm as " $3 R s+3$ " in that it advocates extending animal welfare into the social sphere, beyond the Three Rs of animal research, to include respect for both the knowledge gained to date and for the animals (i.e., species) that were the subjects of study; reciprocal translation of animal research findings into veterinary care; and responsibility to engage the corpus of extant and new knowledge more broadly and to reflect on, reform, and guide constructs, values, and actions of individuals and communities. See Loveless S. Welfare and ethics in veterinary shelter medicine. Today's Veterinary Practice 2011;1(1):50-5.

73. See here, e.g., note 56, Ingensiep 2010, and Berns GS, Brooks AM, Spivak M. Functional MRI in awake unrestrained dogs. PLOS ONE 2012;7(5):e38027. doi:10.1371/journal.pone.0038027.

74. Nivens J. Reckoning with the Beast: Animals, Pain and Humanity in the Victorian Mind. Baltimore, MD: Johns Hopkins University Press; 1972.

75. Nattrass K. "Und die Tiere": Constitutional protection for Germany's animals. Animal Law 2004;10:283-312.

76. Sax B. Animals in the Third Reich: Pets, Scapegoats, and the Holocaust. New York: Continuum; 2000.

77. Köpf P. Ein Herz für Tiere. Hamburg: Verlag JHW Dietz; 1996.

78. "The state protects, in the interest of future generations, the natural basis of life and the animals within the framework of constitutional laws and through the making of laws, and in accordance with ordinances and through judicial decision." See note 75, Nattrass 2004, for a complete review.

79. German Animal Protection League (Deutscher Tierschutzbund). Tierschutz ins GrundgesetzLanger Atem führt zum Erfolg-Konsequente Umsetzung angemahnt-Verbandsklagerecht für Tierschutzorganisationen nächstes Etappenziel [press release]; 2002 May 17; available at http:/ / www. tierschutzbund.de/aktuell/presse/index.htm (last accessed 20 June 2012).

80. See note 75, Nattrass 2004.

81. Meyer C. Animal Welfare Legislation in Canada and Germany: A Comparison. Stuttgart: Peter Lang; 1996.

82. For example, the United States Animal Welfare Act, 7 U.S.C. § 2131 et seq. (1966), regulates commercial and research activities, and-unlike the Tierschutzgesetz-protects only vertebrate species, excluding rats, mice, and birds.

83. Treaty of Lisbon; available at http://europea.eu/lisbon_treaty/full_tedt/index_en.htm (last accessed 20 June 2012). 